\title{
Comparative Wear Characteristics of Some Modern Dental Materials due to the Main Physical Characteristics during the Masticatory Process
}

\author{
Beshtau A Kudzaev ${ }^{1}$, Anna A Remizova², Rita V Kalagova ${ }^{3}$
}

\begin{abstract}
Aim and objective: To design a simple low weight and energy consumption chewing machine, able to implement main characteristics of the masticatory process during testing and study some modern materials from various groups: polymers, composites, and metals.

Materials and methods: A chewing machine that allowed imitating to a high degree of approximation to the real masticatory process in an abrasive medium the movement of test dental crowns made of polymers PEEK and PMMA, metals $\mathrm{ZrO}_{2}$ and Zirconia Prettau, and composites Trinia and Ambarino was designed for research. Dental crowns made of the test material were glued to incisal pins, which were screwed into the rods fixed on the carriage. The lower rods with the attached incisal pins and dental crowns were placed in a container with abrasive material. The carriage, alongside the rods and incisal pins with crowns, performed the reciprocating motion, for which a slider-crank mechanism with an electric drive was used. The press force on the test samples during each cycle was controlled using strain-gauge instrumentation and recorded in the computer memory.

Results: It was found that at the end of the study, the difference between relative changes before and after the experiment in the weight of dental crowns made of Trinia composite was 5.32\% less than dental crowns made of Ambarino; PMMA polymer was $6.057 \%$ less than dental crowns made of PEEK; zirconium dioxide $\mathrm{ZrO}_{2}$ was $0.46 \%$ less than dental crowns made of Prettau zirconium.

Conclusion: An effective design of low energy consumption and weight chewing machine has been developed, which helped to study the wear resistance of some widely used materials.

Clinical significance: The research findings allow a more reasonable approach to the choice of materials to develop various dental structures. Keywords: Chewing machine, Group of composites trinia and ambarino, Group of metals $\mathrm{ZrO}_{2}$ and Zirkonia Prettau, Group of polymers PEEK and PMMA, Wear resistance of dental materials.
\end{abstract}

World Journal of Dentistry (2021): 10.5005/jp-journals-10015-1855

\section{INTRODUCTION}

The masticatory process itself is one of the most important processes in the human body, the quality of which directly affects health. Many modern studies are devoted to the study of its features and the effect on the body state. ${ }^{1-5}$

In their research, scientists often use chewing machines of well-known companies. In turn, various scientific studies are also performed to improve their designs. Scientists try to increase the number of objects simultaneously tested under the same conditions, as well as more accurately reproduce the load on the test samples, the trajectory of their movement, etc. ${ }^{6}$ However, the known designs of chewing machines are quite cumbersome, and copying the trajectory of a tooth movement in space requires complex devices.

In this regard, developing a chewing machine of a simple low weight and energy consumption design that follows the performance of such characteristics as the number of chewing cycles, the path and speed of the test material in the medium that imitates food, the test material trajectory, the load on it, and studying by its means the wear resistance of some popular dental materials is relevant.

To implement the above-mentioned requirements in the machine design, the slider-crank mechanism formed the basis for the machine design. The choice of this mechanism is explained by the following.
${ }^{1}$ Department of Dentistry No. 3, North-Ossetian State Medical Academy, Vladikavkaz, North Ossetia-Alania Republic, Russian Federation

${ }^{2}$ Department of Dentistry No. 3, North-Ossetian State Medical Academy, Vladikavkaz, North Ossetia-Alania, Russian Federation

${ }^{3}$ Department of Chemistry and Physics, North-Ossetian State Medical Academy, Vladikavkaz, North Ossetia-Alania, Russian Federation

Corresponding Author: Rita V Kalagova, Department of Chemistry and Physics, North-Ossetian State Medical Academy, Vladikavkaz, North Ossetia-Alania, Russian Federation, Phone: +79888752790, e-mail:kudzaevaF@yandex.ru

How to cite this article: Kudzaev BA, Remizova AA, Kalagova RV. Comparative Wear Characteristics of Some Modern Dental Materials due to the Main Physical Characteristics during the Masticatory Process. World J Dent 2021;12(5):357-362.

Source of support: Nil

Conflict of interest: None

- In most cases, the lower human jaw makes a movement characterized by a change in the direction of movement near the lowest and highest positions. At that, the movement of the lower jaw, and hence teeth is accelerated at the beginning of one chewing cycle and decelerated at the end of the chewing cycle. Based on the above, such a movement can be considered harmonic. 
- One of the mechanisms that implement harmonic movement is the slider-crank mechanism of a simple design, which has been studied quite deeply theoretically and experimentally and is widely used in a variety of devices.

- When the slider-crank mechanism is in operation, the slider can develop a strong force near the limiting positions, the value of which is easily calculated by the known mechanics formulas. The strong force of the slider at the end of the movement is very similar to the teeth force on the food while chewing it.

Thus, by fixing incisal pins with dental crowns made from the test material on the slider of the crank mechanism and placing them in the container with an imitating food medium, we can sufficiently close to reality imitate the dental crowns movement in the oral cavity during chewing food and the impact on them.

If to determine the force change over time and show it as a graph (Fig. 1) (oscillogram) we can not only control the load during chewing process and the quantity of chewing cycles but also approximately calculate energy amounts, spent on crowns wear process during one cycle and the whole experiment.

In our opinion, the analysis of energy consumption per wear of a unit of dental crown weight can significantly complement the characteristics of the test materials.

However, for a high-quality calculation of energy consumption during each cycle, it is desirable to record with high accuracy the amount of dental crowns movement and the force acting on them by the food imitator. This requires the installation of at least one additional sensor on the designed machine and the related software for the automated calculation of energy consumption.

Consequently, at the first stage of our work, we limited ourselves to designing a basic chewing machine prototype and testing several popular dental materials without regard to the energy consumption per wear both total and calculated.

The study of popular dental materials using the proposed chewing machine can significantly supplement their scientific database and, ultimately, assist the practitioner to choose the most appropriate material in each specific clinical case.

Nowadays, much attention is paid to the study of plastic materials such as PEEK and PMMA and at the same time, such an important property of materials as wear resistance..$^{7-9}$

Data on the wear resistance of such popular materials as $\mathrm{ZrO}_{2}$ and Zirconia Prettau are also of great interest. These materials are studied in many papers, which show specific important properties

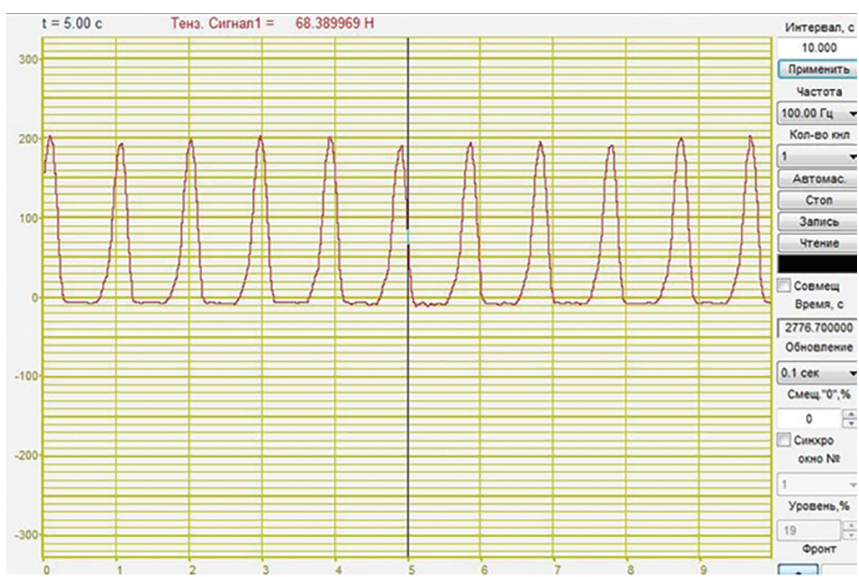

Fig. 1: Graph of the force acting on the crown in time, shown on the computer screen during the experiment that are of great importance for their application in clinical practice. $^{10-12}$

The next group of widely studied dental materials is composites, such as Trinia and Ambarino. Many scientific papers are devoted to the study of their various mechanical characteristics. Researchers have studied such important characteristics as bending modulus, water absorption, stress distribution in prostheses, etc. ${ }^{13-15}$

The following are the description of the proposed chewing machine design and the research findings on the wear resistance of the above popular dental materials.

\section{Materials and Methods}

\section{Substantiation of Some Provisions of Methodology of the Study}

The research methodology was developed based on the results and provisions given in various scientific papers on this issue and regulatory documents. Special attention was paid to the analysis of the international standard provisions ISO/TS 14569-2:2001 “Dental materials-Guidance on testing of wear-Part 2: Wear by two-and/or three body contact" and the Russian State Standard P 55651-2013 by the same name. The Russian State Standard is based on ISO/TS 14569-2:2001 and additionally contains Part 12, which provides a methodology for studying the wear resistance of dental materials, developed by specialists of the Central Research Institute of Dentistry (Moscow).

Based on studying these documents and references given in them, we identified several provisions that must have been considered while developing the methodology of the forthcoming research, as well as in the chewing machine design.

- Currently, there is no single methodology to test dental materials wear and main methods differ significantly. In addition, the polyacrylate and sintered alumina used as the reference material do not reflect the full reality for the antagonist.

- A detailed analysis of methods found that in some of them the authors seek to realize the harmonic movement of the test object (method-Minnesota). However, at that, the movement parameters close to the real ones are not always observed. For example, in the Minnesota method, the applied frequency of the test object movement $4 \mathrm{~Hz}$ is higher than the real one.

Based on paragraph 1, we need to assume that to aggravate the test conditions for materials we can apply a different medium in which more intensive wear will occur.

\section{Preliminary Experiments}

As we see in paragraph 2, some scientists share the view regarding the usefulness of using the harmonic movement by the test object. To finally verify the usefulness of implementing harmonic movement in the machine design, we performed a little preliminary experiment during which a frontal video recording of the food chewing process by five participants of the experiment was made. Then, while viewing the video frame-by-frame, the value of the menton path was measured and graphs were plotted in the time-movement coordinates $[t(c)-S(\mathrm{~mm})]$. The graphs analysis convincingly showed the usefulness of implementing the harmonic movement of the test object in the designed chewing machine.

The following is a description of the proposed small-sized chewing machine that implements the marked provisions. 


\section{Construction of Chewing Machine}

The design is shown in Figure 2. The machine consists of a frame 1 , in the upper part of which an electric motor 2 connected to the reduction gear 3 is fixed. The electric motor is plugged into the network via a transformer 4 . The crank 5 pivotally connected with the connecting rod 6 is fixed to the reducer output shaft 3 . The lower end of the connecting rod is pivotally connected with the slide 7 , to the sides of which wheels resting on the guide frame 1 are fixed. In the lower part of the slide 7 , there are bushings where the rods 7 are fixed, which length can be adjusted by screwing-in or out the bushings.

Incisal pins with fixed dental crowns 9 made of the test materials are screwed in the lower rods 8 . The lower rods 8 with dental crowns 9 are immersed into a metal container 10 filled with material 11 that imitates coarse food. In dental studies, various materials are used as the food imitator, but we have chosen fine-grained quartz sand. Quartz sand is a good abrasive medium, which also has good flowability. While moving the dental crowns up, the sand fills the released space well, i.e., when the dental crowns start moving down, they are already surrounded by the abrasive medium.

Container 10 filled with quartz sand 11 was placed on a steel plate 12. For the plate can restore its shape, it is made of high-carbon steel and is subject to hardening. Shock absorbers 13 made of hard rubber are placed between plate 12 and container 11 , as well as between plate 12 and frame 1 .

Strain sensors 14 connected by wires via an analog-to-digital converter (ADC) 15, signal amplifier 16, laptop 17 are attached on the lower plate side 12 . To control the number of cycles, the unit is with a sensor 18 .

The computer was equipped with a Zetlab software package that ran the "Strain sensor" and "Oscilloscope" programs.

\section{Working Process of Chewing Machine}

The chewing machine operates as follows. While turning on the transformer switch 4, the electric motor 2 starts to rotate, driving the crank 5 fixed to the reducer output shaft 3 . The crank rotation frequency is $60 \mathrm{cpm}$, which equals the average frequency of human mastication.

The crank 5, by the connecting rod 6, drives slide 7 containing fixed rods 8 with dental crowns 9 , which perform a reciprocating motion. The crank length is $5 \mathrm{~mm}$, i.e., the motion of dental crowns

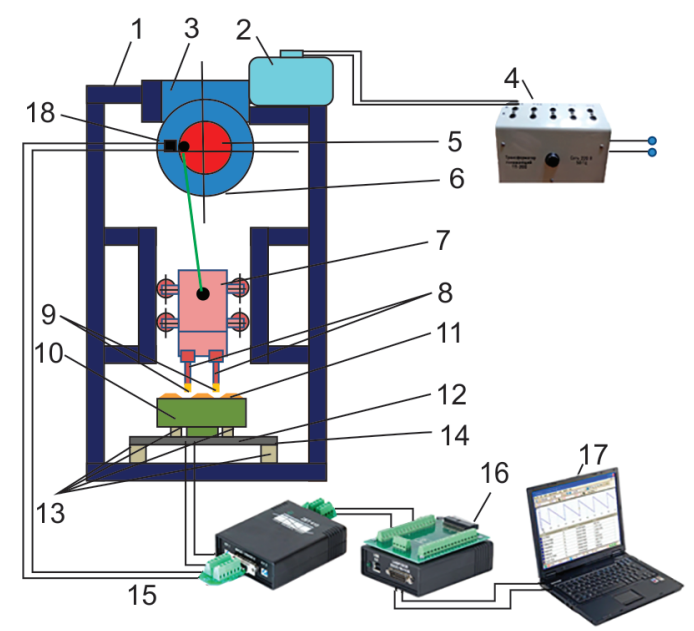

Fig. 2: Design of the proposed chewing machine in the abrasive material is $10 \mathrm{~mm}$, which is close to the actual path of tooth 4.6 in the oral cavity during the food chewing.

When the dental crowns 9 are immersed in the abrasive material 11 , the latter is compressed and the sensor signal 14 is displayed on the computer screen 17.

\section{Research Conditions}

The number of depth cycles for each batch of test crowns was assumed to be $1.5 \times 10^{5}$.

The dental crown depth force was assumed to be $200 \mathrm{~N}$. Since two rods with crowns are placed simultaneously in the slide, the force on each was $100 \mathrm{~N}$.

The force was adjusted by changing the length of the connecting rod 6 and rods 8 , and in the second machine designby displacing the plate 12 on threaded supports. The uniform distribution of force on each crown was provided by changing the length of the rods 8 and rubber shock absorbers 13 .

The speed of the dental crowns movement in the abrasive material changed according to the harmonic law and was calculated by the formulas used in the technology. Its maximum value was approximately $0.03 \mathrm{~m} / \mathrm{s}$, the minimum is 0 .

The dental crowns heating temperature was controlled by a pyrometer CEM DT 8865.

If the lower slide wheels 7 are placed with a gap up to $0.5 \mathrm{~mm}$, this helped to imitate the jaw lateral displacement up to $1.5 \mathrm{~mm}$ and, accordingly, the tooth during the food chewing. That is, it was possible to achieve not only the test dental crowns up or down displacement but also offset at maximum depth, i.e., at the end of the abrasive material compression.

The described design features of the chewing machine, which consist of the use of a crank mechanism, eventually provided small values of the drive energy intensity and weight. The machine motor power is $6 \mathrm{~W}$, the machine weight (without a step-down transformer and measuring equipment) is $7.8 \mathrm{~kg}$.

\section{Machine Calibration}

All standard measuring equipment that is used in the research was checked beforehand in a specialized laboratory. Only then, the final machine calibration was performed.

During the machine operation, the "Strain sensor" and "Oscilloscope" programs ran on the laptop to measure the force acting on the dental crowns made of the test material. Their force value was deduced in ADC units, which then need to be converted to Newtons. For the force to be expressed in Newtons at once, in tab "Parameters" of the "Strain sensor" program, we entered the gain value, which was determined experimentally.

To achieve that, we made a stand, which was a high rectangular frame. The distance between the vertical frame sides was equal to the distance between the lower rubber shock absorbers 13 (Fig. 2). Two horizontal brackets were attached to the vertical rectangle sides on which the lower rubber shock absorbers 13 and the plate 12 with glued sensors 14 were installed. Top shock absorbers 13 , which fixed the plate, were installed on top of the plate 12 . The hanging down frame was placed in the center of the plate. Loads of various sizes were suspended from the frame.

The calibration was performed as follows. The measuring equipment with a laptop was connected to plate 12, the software was run, and all the equipment and sensors were warmed up for 20 minutes. After the equipment was warmed up, a mass of $0.5 \mathrm{~kg}$ was suspended from hanging down the frame. Multiplying this value by 9.81 we have obtained the weight value in Newton's. In the 
"Strain sensor" program window, the weight value was expressed in ADC units. The results were tabulated. Then, the mass $0.5 \mathrm{~kg}$ more than the previous one was suspended and the corresponding ADC readings were taken. The experiments were performed to a mass value of $40 \mathrm{~kg}$.

The table data were processed by mathematical methods of statistics. To this end, the ratio $k_{\mathrm{i}}=G_{\mathrm{i}} / A_{\mathrm{i}}$ was found for each pair of weight values $\left(G_{\mathrm{i}}\right)$ and $\operatorname{ADC}$ units $\left(A_{\mathrm{i}}\right)$, i.e., the gain value for each experiment. After that, the arithmetic mean of $k_{\mathrm{i}}$, variance, mean square deviation, the coefficient of variation, and the relative arithmetic mean error were found. According to the processing results, its value was $2.98 \%$.

The calculated value of the arithmetic mean gain of $k_{m}$ is the unknown value.

The significance of discrepancies between the calculated force values and the experimental ones was checked using Pearson's criterion. Based on the calculation results, the set gain value provided reliable results.

Further, shock absorbers 13 (Fig. 2) with the measuring plate 12 were installed back on the frame 1 of the chewing machine. The machine is calibrated.

\section{Test Objects}

We selected popular materials from three groups for research: (1) polymers-PEEK and PMMA; (2) composites Trinia and Ambarino; (3) metals zirconium dioxide $\mathrm{ZrO}_{2}$ and Prettau Zirconium.

Initially, a wax dental crown was modeled with an anatomic occlusal surface of tooth 4.5, and the contact, buccal and lingual surfaces were cylindrical. Then, the dental crowns were milled by CAD/CAM equipment.

Three dental crowns were made from each material.

Further, the dental crowns were attached to specially sharpened and milled incisal pins (Fig. 3) using dual-cure glass ionomer cement Relix U 200, through which they were subsequently attached to adjustable rods 8 (Fig. 2). Figure 3 is shown a pin with a crown ready to be fastened on machine. As you can see in Figure 3, the fastening part of the pin does not need high precision of production.

\section{Abrasive Material}

Quartz sand with a density of $1.2 \mathrm{~g} / \mathrm{cm}^{3}$ served as an abrasive material.

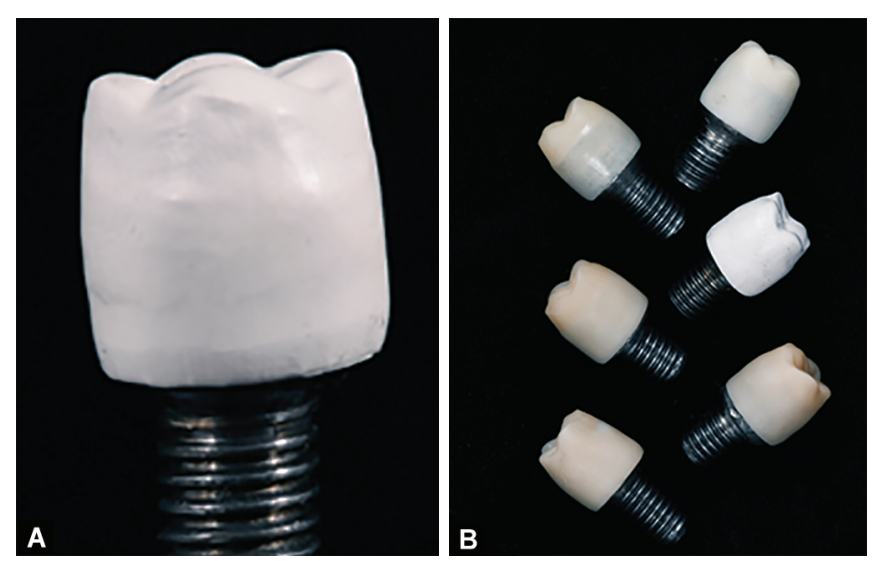

Figs $3 A$ and B: General view of the samples fixed of incisal pins. (A) Dental crown fixed on the incisal pin; (B) One of the dental crown batches prepared for research

\section{Measurements}

During studies, the linear dimensions of the crowns and their weight before and after the experiment were measured.

To study the linear dimensions, the crown diameters were measured in two mutually perpendicular directions using a micrometer MK 0-25.

The dental crowns weight was determined by OHAUS Pioneer analytical scales.

\section{Processing of Measurement Results}

The measurement results were processed by of Mathematical Methods of Statistics. The following values were determined: the arithmetic mean value, variance, standard deviation, coefficient of variation, arithmetic mean error, and relative arithmetic mean error.

\section{Results}

The research has found that the developed chewing machine provides the stability of the specified load on the test dental crowns and the number of depth cycles in the abrasive medium. This is evident from the oscillogram recorded during the test (Fig. 1). It can also be mentioned on the Figure 1 that machine provides stability of stress on the crowns, and, that is very important, identity of the process of loading.

Measurement of the test dental crowns diameter has shown that in their lower part, close to the cusp, there was a slight increase in diameter. In our opinion, this is due to the flat surface of the bottom in the container with the abrasive material with many dental crown action cycles. Since the values of the diameter changes in our case were not very informative, they were excluded from the further analysis.

Data on changes in the weight of the test samples are of the highest value. Its average measurement values and, accordingly, the average values of changes are shown in the Table 1.

It should be noted that the incisal pins themselves were not strictly required to have the same weight. This is because the dental crown surface completely covered the cylindrical part of the incisal pin and its threaded part was screwed into the body of the adjustable rod 8 (Fig. 2) and was not subject to wear. Thus, the difference between the values in column 3 of the table and the corresponding values in column 4 shows us the absolute value of the dental crown wear given in column 5 .

As shown in Table 1, the absolute values of the weight of the crown in the same group, given in column 5, differ greatly. Due to the difference in the initial weight values (column 2), it might be concluded that the highest value from the informative point of view is represented by the relative change values in the dental crowns weight, which is shown in column 6 . It is convenient to show these values in diagram form, which greatly facilitates to analyze the research findings (Fig. 4).

It would be incorrect to compare the wear of materials from such different groups if the experimental conditions were not so strict, so a brief review of the wear findings between different groups can contribute to a more complete picture of the test materials.

As the Table 1 and Figure 4 show, materials in group IIcomposites were the most wear-prone. The weight loss of the test materials in this group reached $22.33 \%$ of the initial, which, of course, is a lot.

Materials in group I-polymers that lose weight on average up to $15.13 \%$ of the initial were significantly less wear-prone. 
Table 1: Average measurement values of the test samples weight and average values of its change

\begin{tabular}{|c|c|c|c|c|c|c|}
\hline \multirow[b]{3}{*}{ Group } & \multirow[b]{3}{*}{ Material } & \multicolumn{3}{|c|}{ Weighted average value of a dental crown } & \multicolumn{2}{|c|}{$\begin{array}{c}\text { Changes in the weighted average value } \\
\text { of a dental crown }\end{array}$} \\
\hline & & \multicolumn{2}{|c|}{ Before test } & \multirow{2}{*}{$\begin{array}{l}\text { After test } \\
\text { With incisal pin, } \\
m_{k s 1}, g\end{array}$} & \multirow[b]{2}{*}{ Absolute $\Delta m_{a^{\prime}} g$} & \multirow[b]{2}{*}{ Relative $\Delta m_{\alpha} \%$} \\
\hline & & $\begin{array}{l}\text { Without incisal pin } \\
m_{k^{\prime}} g\end{array}$ & $\begin{array}{l}\text { With incisal pin, } \\
m_{k k^{\prime}} g\end{array}$ & & & \\
\hline 0 & 1 & 2 & 3 & 4 & 5 & 6 \\
\hline \multirow[t]{2}{*}{1} & PEEK & 0.4982 & 5.1 & 5.0548 & 0.0452 & 9.073 \\
\hline & PMMA & 0.4393 & 4.6702 & 4.6037 & 0.0665 & 15.13 \\
\hline \multirow[t]{2}{*}{2} & Trinia & 0.5549 & 5.2241 & 51297 & 0.0944 & 17.01 \\
\hline & Ambarino & 0.7442 & 5.4691 & 5.3029 & 0.1662 & 22.33 \\
\hline \multirow[t]{2}{*}{3} & $\mathrm{ZrO}_{2}$ & 1.9737 & 6.6214 & 6.6115 & 0.0099 & 0.5 \\
\hline & Zirconia Prettau & 1.9289 & 6.575 & 6.5565 & 0.0185 & 0.96 \\
\hline
\end{tabular}

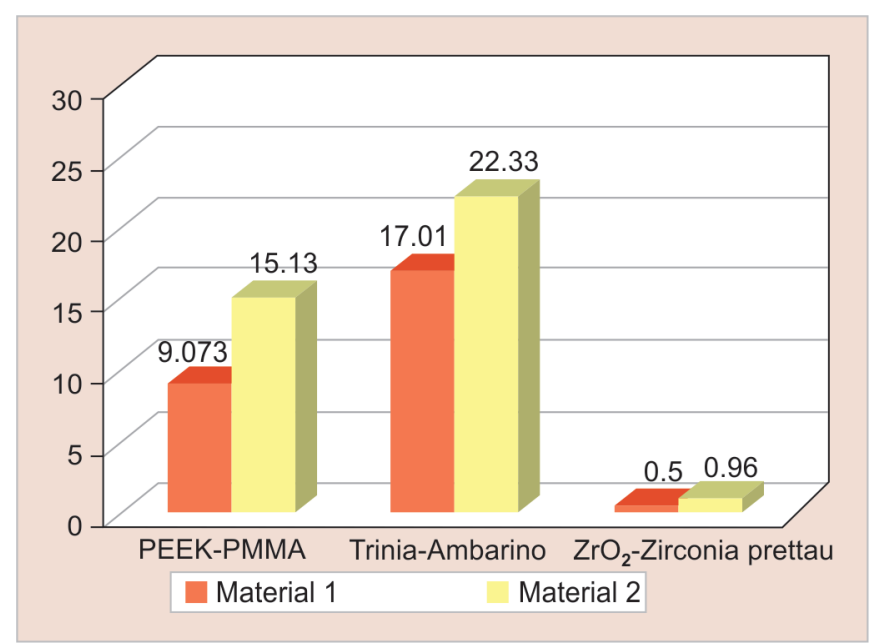

Fig. 4: Bar graph of average values of the relative change in the weight of the test materials (\%) by groups

Materials in group III-metals were the least wear-prone. The maximum weight reduction value did not exceed $0.96 \%$ of the crown weight value before the test.

The dental crowns heating temperature during the test did not exceed $55^{\circ} \mathrm{C}$.

The analysis of the crown surfaces using an optical magnification device did not reveal any cases of their chipping or visible cracks, which allowed a positive characterization of all test materials.

\section{Discussion}

A more detailed study of the research findings allows stating as follows. The materials of each group had a significant difference in their wear resistance, as shown in the Table 1 and Figure 4. Under the strict conditions of our experiment, the weight loss of the Trinia composite was 5.32\% less than Ambarino, which is very significant. Moreover, dental crowns made of Ambarino had the largest weight loss values in absolute units- $0.1662 \mathrm{~g}$.

Thus, the dental material Trinia, which is characterized not only by good wear resistance and hardness due to multi-directional fiberglass but also by lightness, is obviously more preferable to make designs requiring these materials to have both significant technological and esthetic properties. For example, overdenture supported by implants. Ambarino, wear resistance of which is somewhat lower, also combining high esthetic properties and the Chameleon effect, is advisable to be used for manufacturing dental crowns, inlays, veneers, and prosthetic bridges.

As the research findings show significant differences in the wear degree were observed among dental crowns made of polymers $-6.057 \%$, but in absolute units, the weight losses were less than in the group of composites and ranged between 0.0452 and $0.0665 \mathrm{~g}$.

The revealed difference in the wear resistance of materials of this group is not significant to make temporary dental designs, which, in principle, is one of the main recommendations to use these materials. To manufacture a temporary dental crown, it is obviously preferable to use PMMA, since the designs made of it are more esthetic. PEEK is good for making designs that require no special esthetics but need hardness (e.g., temporary abutments).

Dental crowns made of the test metals were the least wearprone under the strict conditions of our experiment, and the minimum weight loss values were in crowns made of zirconium dioxide $\mathrm{ZrO}_{2}-0.0099 \mathrm{~g}$, which was $0.5 \%$ of the initial value of the crown weight. Crowns made of Zirkonia Prettau had a slightly higher weight loss value-on average $0.0185 \mathrm{~g}$ or $0.96 \%$ of the initial crown weight value.

Differences in the wear resistance of the test materials from the group of metals are not very significant that also affect the choice of designs, which is largely based on their esthetic properties. For example, Zirkonia Prettau is preferable to be used to make designs for the front teeth due to its greater whiteness and transparency, and $\mathrm{ZrO}_{2}$ - for the masticatory teeth.

The data obtained on the wear resistance of the test materials are difficult to compare with the results obtained by other scientists due to differences in the experimental methods. ${ }^{16-20}$ This is primarily a type of abrasive material, as well as differences in kinematic parameters of the test object movement and loads on it.

However, the results of this experiment, performed under the same, well-controlled conditions, significantly supplement the available data characterizing dental crowns made of these materials.

In dental science, a large range of studies deals with the comparative wear resistance of tooth enamel and dental materials during their interaction with abrasive, tooth enamel wear while interacting with dental materials, dental materials wear while interacting with tooth enamel, etc. ${ }^{21-23}$ Many of these experiments are performed by scientists using chewing machines that load the 
test object in cycles. The proposed design of the chewing machine allows implementation of some of the users in these methods parameters of the test objects movement.

To implement experiments in which the tooth moves along the dental material surface acting on it with a certain force, the chewing machine design can be upgraded with additional brackets that will allow plate 12 (Fig. 2) place vertically and the rods 8 with incisal pins 9 horizontally on the carriage 7.

When the machine is equipped with a carriage movement sensor, it is possible to calculate the energy consumption per the test material wear.

The proposed machine can serve as a basic module to design a more high-capacity machine containing the desired number of sections.

As we see, the designed sample of the chewing machine is small-sized, has low weight, low cost, is easy to manufacture, sufficiently implements the main characteristics of the masticatory process, and has large reserves for further improvement.

The experiment had limitations due to lack of saliva imitator in the material, simulating food and there were not used natural teeth during the study as well. With these conditions, it will be possible to get information simultaneously about the wear resistance of dental materials and natural teeth, so it is really appropriate.

\section{CONCLUSION}

The effective design of the chewing machine that simulates the masticatory process with a high similarity, having low energy consumption and weight has been developed, which helped to study the wear resistance of materials in three groups: polymers, composites, and metals.

\section{ACKNOWLedgments}

The authors thank the Vice-rector for Science of Gorsky State Agrarian University, Dr Sci. Tech., Professor A.B. Kudzaev for valuable advice on technical issues that arose during the development of the chewing machine.

\section{References}

1. Takei E, Kulvaanich S, Tsujimura T, et al. Age-related changes in functional adaptation to bolus characteristics during chewing. Physiol Behav 2020;225:113102. DOI: 10.1016/j.physbeh.2020.113102.

2. Kugimiya $Y$, Watanabe $Y$, Shirobe $M$, et al. A comparison of colorimetric and visual methods for the assessment of masticatory performance with color-changeable chewing gum in older persons. J Dent Sci 2020(1):In press, corrected proof, Available online 10.1016/j. jds.2020.08.010.

3. Kassim S. The impact of protective psychosocial factors on khat chewing among male medical and dental future health-care providers in Yemen. 2020(4):In press, corrected proof, Available online 10.1016/j.jds.2019.08.009.

4. Al-Duais MA, Al-Awthan YS. Association between qat chewing and dyslipidaemia among young males. J Taibah Univers Med Sci 2019;14(6):538-546. DOI: 10.1016/j.jtumed.2019.09.008.

5. Miguel-Kergoat S, Azais-Braesco V, Burton-Freeman B, et al. Effects of chewing on appetite, food intake and gut hormones: A systematic review and meta-analysis. Physiol Behav 2015;151:88-96. DOI: 10.1016/j.physbeh.2015.07.017.
6. Xu WL, Lewis D, Bronlund JE, et al. Mechanism, design and motion control of linkage chewing device for food evalution. Mechan Machine Theory 2008;43(3):376-389. DOI: 10.1016/j. mechmachtheory.2007.03.004.

7. Wimmer T, Mildred A, Huffmann O, et al. Two-body wear rate of PEEK, CAD/CAM resin composite and PMMA: Effect of specimen geometries, antagonist materials and test set-up configuration. Dent Mat 2016;32(6):e127-e136. DOI: 10.1016/j.dental.2016.03.005.

8. Avanzini A, Dnzella G, Mazzu A, et al. Wear and rolling contact fatigue of PEEK and PEEK composites. Tribol Int 2013;57:22-30. DOI: 10.1016/j. triboint.2012.07.007.

9. Liu Y, Song S, Timmers H. Correlation of polymer wear-debris generation between microscratching and macroscopic wear. Tribol Int 2016;93(part A):202-213. DOI: 10.1016/j.triboint.2015. 09.010 .

10. Kwon M-S, Oh S-Y, Cho S-A. Two-body wear comparison of zirconia crown, gold crown, and enamel against zirconia. J Mechan Behav Biomed Mat 2015;47:21-28. DOI: 10.1016/j.jmbbm.2014.11.029.

11. Kim M-J, Oh S-H, Kim J-H, et al. Wear evaluation of the human enamel opposing different Y-TZP dental ceramics and other porcelains. J Dentis 2012;40(11):979-988. DOI: 10.1016/j.jdent.2012.08.004.

12. Zhang F, Spies BC, Vleugels J, et al. High-translucent yttria-stabilized zirconia ceramics are wear-resistant and antagonist-friendly. Dent Mat 2019;35(12):1776-1790. DOI: 10.1016/j.dental.2019.10.009.

13. Suzaki N, Yamaguchi S, Hirose N, et al. Evaluation of physical properties of fiber-reinforced composite resin. Dent Mat 2020;36(8):987-996. DOI: 10.1016/j.dental.2020.04.012.

14. Zaparolli D, Peixoto RF, Pupim D, et al. Photoelastic analysis of mandibular full-arch implant-supported fixed dentures made with different bar materials and manufacturing techniques. Mat Sci Engineer: C 2017;81:144-147. DOI: 10.1016/j.msec.2017.07.052.

15. Flury S, Diebold E, Peutzfeldt A, et al. Effect of artificial toothbrushing and water storage on the surface roughness and micromechanical properties of tooth-colored CAD-CAM materials. J Prosthe Detis 2017;117(6):767-774. DOI: 10.1016/j.prosdent.2016.08.034.

16. D'Arcangelo C, Vanini L, Rondoni GD, et al. Wear evalution of prosthetic materials opposing themselves. Operat Dentis 2018;43(1):38-50. DOI: 10.2341/16-212-L.

17. Hadi MR, Al-masoody AH, Bager Osamah A-M, et al. Evaluation of abrasive wear resistance of polymethylmethacrylate material reinforced with zirconium and dental stone. Drug Invention Today 2019;11(11):3081-3084.

18. Khare N, Limaye PK, Soni NL, et al. Friction and wear characteristics of PEEK and PEEK composites in water lubricated slow speed sliding. Tribol Online 2015;10(1):1881-2198. DOI: 10.2474/trol.10.84.

19. Melk L, Rovira JJ, Antti M-L, et al. Coefficient of friction and wear resistance of zirconia-MWCNTs composites. Ceram Int 2015;41(1):459468. DOI: 10.1016/j.ceramint.2014.08.092.

20. Emre G, Akkus A, Karamis MB. Wear resistance of polymethyl methacrylate (PMMA) with the addition of bone ash, hydroxylapatite and keratin. IOP Conf Ser Mater Sci Engineer 2018;295(1):012004. DOI: 10.1088/1757-899X/295/1/012004.

21. D'Arcangelo C, Vanini L, Rondoni GD, et al. Wear properties of dental ceramics and porcelains compared with human enamel. J Prosthe Dentis 2016;115(3):350-355. DOI: 10.1016/j.prosdent.2015. 09.010 .

22. Zandparsa R, Hiravama H, Jonson MI. Effect of different dental ceramic systems on the wear of human enamel: An in vitro study. J Prosthe Dentis 2016;115(2):230-237. DOI: 10.1016/j.prosdent.2015.09.005.

23. Sripetchdanond J, Leevailoj C. Wear of human enamel opposing monolithic zirconia, glass ceramic, and composite resin: an in vitro study. J Prosthe Dentis 112(5):1141-1150. DOI: 10.1016/j. prosdent.2014.05.006. 